\title{
Exact solvability and quantum integrability of a derivative nonlinear Schrödinger model
}

\author{
B. BASU-Mallick And TANaya Bhattacharyya
}

Theory Group, Saha Institute of Nuclear Physics, 1/AF Bidhan Nagar, Kolkata 700064 , India

Received XXX

\begin{abstract}
By using a variant of quantum inverse scattering method (QISM) which is directly applicable to field theoretical systems, we derive all possible commutation relations among the operator valued elements of the monodromy matrix associated with an integrable derivative nonlinear Schrödinger (DNLS) model. From these commutation relations we obtain the exact Bethe eigenstates for the quantum conserved quantities of DNLS model. We also explicitly construct the first few quantum conserved quantities including the Hamiltonian in terms of the basic field operators of this model. It turns out that this quantum Hamiltonian has a new kind of coupling constant which is quite different from the classical one. This fact allows us to apply QISM to generate the spectrum of quantum DNLS Hamiltonian for the full range of its coupling constant.
\end{abstract}

Key words: Derivative nonlinear Schrödinger model, Yang-Baxter equation, Quantum conserved quantities

\section{Introduction}

Derivative nonlinear Schrödinger (DNLS) model in $1+1$-dimension is a wellknown integrable system which has found applications in different areas of physics like circularly polarized nonlinear Alfven waves in a plasma [1], quantum properties of solitons in optical fibres [2] and some chiral Luttinger liquids [3]. The equation of motion for the Chen-Lee-Liu type classical DNLS model is given by

$$
i \partial_{t} \psi(x, t)+\partial_{x x} \psi(x, t)-4 i \xi \psi^{*}(x, t) \psi(x, t) \partial_{x} \psi(x, t)=0,
$$

where $\partial_{t} \equiv \frac{\partial}{\partial t}, \partial_{x} \equiv \frac{\partial}{\partial x}, \partial_{x x} \equiv \frac{\partial^{2}}{\partial x^{2}}$ and $\xi$ is a real parameter representing the strength of the nonlinear interaction term. The Hamiltonian

$$
H=\int_{-\infty}^{+\infty}\left\{-\psi^{*}(x) \partial_{x x} \psi(x)+i \xi \psi^{* 2}(x) \partial_{x} \psi^{2}(x)\right\} d x,
$$

and an equal time Poisson bracket structure given by $\{\psi(x), \psi(y)\}=\left\{\psi^{*}(x), \psi^{*}(y)\right\}$ $=0,\left\{\psi(x), \psi^{*}(y)\right\}=-i \delta(x-y)$, generate eqn.(11) as a canonical evolution. By using the Lax operator associated with this DNLS model, one can explicitly construct its conserved quantities in a recursive way [4]. It can be shown that such infinite number of conserved quantities yield vanishing Poisson bracket relations among 
themselves. This fact establishes the classical integrability of DNLS model (1) in the Liouville sense.

In this review article, our aim is to study the spectrum for the quantum version of the above mentioned DNLS model [5] and discuss the nature of the related conserved quantities [6]. In Sec.2, we construct the Bethe eigenstates for these conserved quantities by using a variant of quantum inverse scattering method (QISM) [7] which is directly applicable to continuum field models. In Sec.3, we derive the expressions of the first few quantum conserved quantities including the Hamiltonian in terms of the basic field operators of the system. Sec.4 is the concluding section.

\section{Application of QISM to quantum DNLS model}

In the quantized version of DNLS model, the basic field operators satisfy equal time commutation relations given by

$$
[\psi(x), \psi(y)]=\left[\psi^{\dagger}(x), \psi^{\dagger}(y)\right]=0, \quad\left[\psi(x), \psi^{\dagger}(y)\right]=\hbar \delta(x-y),
$$

$\hbar$ being the Planck's constant, and the vacuum state is defined through the relation: $\psi(x)|0\rangle=0$. For applying QISM [7] to this model, it is necessary to find out at first an appropriate Lax operator which would satisfy quantum Yang-Baxter equation (QYBE) in continuum. Such a quantum Lax operator is obtained as [5]

$$
\mathcal{U}_{q}(x, \lambda)=i\left(\begin{array}{cc}
f \psi^{\dagger}(x) \psi(x)-\lambda^{2} / 4 & \xi \lambda \psi^{\dagger}(x) \\
\lambda \psi(x) & -g \psi^{\dagger}(x) \psi(x)+\lambda^{2} / 4
\end{array}\right)
$$

where $f=\frac{\xi e^{-i \alpha / 2}}{\cos \alpha / 2}, g=\frac{\xi e^{i \alpha / 2}}{\cos \alpha / 2}$ and $\alpha$ is a real parameter which is fixed through the relation: $\sin \alpha=-\hbar \xi$. Due to this relation, it is evident that QISM is applicable to quantum DNLS model when the parameter $\xi$ satisfies a restriction given by $|\xi| \leq \frac{1}{\hbar}$.

Using certain symmetry properties of the quantum Lax operator (3), the corresponding quantum monodromy matrix $\mathcal{T}(\lambda)$ can be written in the form

$$
\mathcal{T}(\lambda)=\left(\begin{array}{cc}
A(\lambda) & -\xi B^{\dagger}(\lambda) \\
B(\lambda) & A^{\dagger}(\lambda)
\end{array}\right)
$$

Expressing QYBE satisfied by the above monodromy matrix in elementwise form, we obtain [5]

$$
\begin{aligned}
& {[A(\lambda), A(\mu)]=0,[B(\lambda), B(\mu)]=0, A(\lambda) B^{\dagger}(\mu)=\frac{\mu^{2} q-\lambda^{2} q^{-1}}{\mu^{2}-\lambda^{2}-i \epsilon} B^{\dagger}(\mu) A(\lambda),} \\
& B(\mu) B^{\dagger}(\lambda)=\tau(\lambda, \mu) B^{\dagger}(\lambda) B(\mu)+4 \pi \hbar \lambda \mu \delta\left(\lambda^{2}-\mu^{2}\right) A^{\dagger}(\lambda) A(\lambda), \quad(5 a, b, c, d)
\end{aligned}
$$

where $\tau(\lambda, \mu)=\left[1+\frac{8 \hbar^{2} \xi^{2} \lambda^{2} \mu^{2}}{\left(\lambda^{2}-\mu^{2}\right)^{2}}-\frac{4 \hbar^{2} \xi^{2} \lambda^{2} \mu^{2}}{\left(\lambda^{2}-\mu^{2}-i \epsilon\right)\left(\lambda^{2}-\mu^{2}+i \epsilon\right)}\right]$. Due to eqn.(5a), it follows that all operator valued coefficients occurring in the expansion of $\ln A(\lambda)$ in powers 
of $\lambda$ must commute among themselves. Consequently, $\ln A(\lambda)$ may be treated as the generator of conserved quantities for the quantum integrable DNLS model. By using algebraic Bethe ansatz, one can also find out the eigenfunctions and eigenvalues corresponding to different expansion coefficients of $\ln A(\lambda)$. However, such eigenvalues would be complex quantities in general. To make the eigenvalues real, we define another operator $\hat{A}(\lambda)$ through the relation: $\hat{A}(\lambda) \equiv A\left(\lambda e^{-\frac{i \alpha}{2}}\right)$ and expand $\ln \hat{A}(\lambda)$ as

$$
\ln \hat{A}(\lambda)=\sum_{n=0}^{\infty} \frac{i \mathcal{C}_{n}}{\lambda^{2 n}} .
$$

With the help of eqns.(5c) and (6), we easily find that

$$
\begin{aligned}
\mathcal{C}_{0}\left|\mu_{1}, \mu_{2}, \cdots, \mu_{N}\right\rangle & =\alpha N\left|\mu_{1}, \mu_{2}, \cdots, \mu_{N}\right\rangle, \\
\mathcal{C}_{n}\left|\mu_{1}, \mu_{2}, \cdots, \mu_{N}\right\rangle & =\frac{2}{n} \sin (\alpha n)\left\{\sum_{j=1}^{N} \mu_{j}^{2 n}\right\}\left|\mu_{1}, \mu_{2}, \cdots, \mu_{N}\right\rangle,
\end{aligned}
$$

where $n \geq 1$ and $\left|\mu_{1}, \mu_{2}, \cdots, \mu_{N}\right\rangle \equiv B^{\dagger}\left(\mu_{1}\right) B^{\dagger}\left(\mu_{2}\right) \cdots B^{\dagger}\left(\mu_{N}\right)|0\rangle$ represent the Bethe eigenstates for all $\mathcal{C}_{n} \mathrm{~s}$. For the case of scattering states, $\mu_{j} \mathrm{~s}$ are chosen as all distinct real or pure imaginary numbers. We can also construct the quantum soliton states or bound states for DNLS model by choosing complex values of $\mu_{j}$ in an appropriate way $[4,5]$. For the case of both scattering and solitonic bound states, eqn.(7) yields real eigenvalues for all $\mathcal{C}_{n} \mathrm{~s}$.

Thus, by applying algebraic Bethe ansatz, we obtain the spectra of all $\mathcal{C}_{n} \mathrm{~s}$ which are formally defined through the expansion (6). Next, we turn our attention to the explicit construction of these quantum conserved quantities in terms of basic field operators like $\psi(x)$ and $\psi^{\dagger}(x)$. In analogy with the cases of nonlinear Schrödinger (NLS) model and sine-Gordon model, one may think that the Hamiltonian of quantum DNLS model can also be obtained as the normal ordered version of the corresponding classical Hamiltonian (2). However, since QYBE restricts the value of $\xi$ as: $|\xi| \leq \frac{1}{\hbar}$, such a quantum Hamiltonian would be solvable by QISM only within this limited range of coupling constant. So at this point, our target is to find out explicit expressions for the first few quantum conserved quantities of DNLS model and investigate whether the above mentioned limitation about the applicability of QISM can be resolved in some way.

\section{Quantum conserved quantities of DNLS model}

For obtaining the quantum conserved quantities of DNLS model in terms of the basic field operators $\psi(x)$ and $\psi^{\dagger}(x)$, here we wish to follow the approach of Ref.8 where first few conserved quantities of the quantum NLS model has been constructed explicitly by using the so called 'fundamental relation'. To this end, we consider Jost solutions associated with the quantum Lax operator (3). It can be 
shown that the components of the Jost solution $\rho(x, \lambda) \equiv\left(\begin{array}{c}\rho_{1}(x, \lambda) \\ \rho_{2}(x, \lambda)\end{array}\right)$, associated with boundary conditions at $x \rightarrow-\infty$, follow the differential equations given by [6]

$$
\begin{aligned}
& \partial_{x} \rho_{1}(x, \lambda)=-\frac{i \lambda^{2}}{4} \rho_{1}(x, \lambda)+i f \psi^{\dagger}(x) \rho_{1}(x, \lambda) \psi(x)+i \xi \lambda \psi^{\dagger}(x) \rho_{2}(x, \lambda), \\
& \partial_{x} \rho_{2}(x, \lambda)=\frac{i \lambda^{2}}{4} \rho_{2}(x, \lambda)-i g \psi^{\dagger}(x) \rho_{2}(x, \lambda) \psi(x)+i \lambda \rho_{1}(x, \lambda) \psi(x) .
\end{aligned}
$$

There exist two independent $\rho(x, \lambda)$ satisfying the above relations with boundary conditions like $\left(\begin{array}{c}\rho_{1}(x, \lambda) \\ \rho_{2}(x, \lambda)\end{array}\right) \stackrel{x \rightarrow-\infty}{\longrightarrow}\left(\begin{array}{c}e^{-i \lambda^{2} x / 4} \\ 0\end{array}\right)$ or $\left(\begin{array}{c}\rho_{1}(x, \lambda) \\ \rho_{2}(x, \lambda)\end{array}\right) \stackrel{x \rightarrow-\infty}{\longrightarrow}\left(\begin{array}{c}0 \\ e^{i \lambda^{2} x / 4}\end{array}\right)$. On the other hand, the components of Jost solution $\tau(x, \lambda) \equiv\left(\begin{array}{c}\tau_{1}(x, \lambda) \\ \tau_{2}(x, \lambda)\end{array}\right)$ associated with the boundary conditions at $x \rightarrow+\infty$ follow the differential equations given by

$$
\begin{aligned}
& \partial_{x} \tau_{1}(x, \lambda)=-\frac{i \lambda^{2}}{4} \tau_{1}(x, \lambda)+i g \psi^{\dagger}(x) \tau_{1}(x, \lambda) \psi(x)+i \xi \lambda \psi^{\dagger}(x) \tau_{2}(x, \lambda), \\
& \partial_{x} \tau_{2}(x, \lambda)=\frac{i \lambda^{2}}{4} \tau_{2}(x, \lambda)-i f \psi^{\dagger}(x) \tau_{2}(x, \lambda) \psi(x)+i \lambda \tau_{1}(x, \lambda) \psi(x) .
\end{aligned}
$$

Again, there exist two independent $\tau(x, \lambda)$ satisfying the above relations and obeying boundary conditions like $\left(\begin{array}{c}\tau_{1}(x, \lambda) \\ \tau_{2}(x, \lambda)\end{array}\right) \stackrel{x \rightarrow+\infty}{\longrightarrow}\left(\begin{array}{c}0 \\ e^{i \lambda^{2} x / 4}\end{array}\right)$ or $\left(\begin{array}{c}\tau_{1}(x, \lambda) \\ \tau_{2}(x, \lambda)\end{array}\right) \stackrel{x \rightarrow+\infty}{\longrightarrow}$ $\left(\begin{array}{c}-e^{-i \lambda^{2} x / 4} \\ 0\end{array}\right)$. It may be noted that the sets of equations (8) and (9), satisfied by the quantum Jost solutions defined through boundary conditions at $x \rightarrow+\infty$ and $x \rightarrow-\infty$ respectively, are not identical in form. This happens due to the fact that, unlike the cases of most other integrable systems, the quantum Lax operator (3) of DNLS model is not a traceless matrix [6].

Next, let us define the quantum Wronskian associated with the Jost solutions $\tau(x, \lambda)$ and $\rho(x, \lambda)$ as

$$
\Lambda_{\rho, \tau}(x, \lambda)=\tau_{2}(x, \lambda) \rho_{1}(x, \lambda)-\tau_{1}(x, \lambda) \rho_{2}(x, \lambda) .
$$

All elements of the quantum monodromy matrix $\mathcal{T}(\lambda)$ (4) can be expressed through this quantum Wronskian by taking different choices of $\rho(x, \lambda)$ and $\tau(x, \lambda)[6]$. Thus the quantum Wronskian (10) does not really depend on the value of the coordinate $x$. In analogy with the quantum Wronskian, we define another operator associated with the Jost solutions of DNLS model as

$$
\Gamma_{\rho, \tau}(x, \lambda)=\tau_{2}(x, \lambda) \rho_{1}(x, \lambda)+\tau_{1}(x, \lambda) \rho_{2}(x, \lambda) .
$$

Next, we propose that, the quantum conserved quantities $\left(I_{n}\right)$ of DNLS model would annihilate the vacuum state and obey the 'fundamental relation' of the form

$$
\left[I_{n}, \Lambda_{\rho, \tau}(\lambda)\right]=\frac{\hbar \lambda^{2 n}}{2^{n+1}}\left\{\Gamma_{\rho, \tau}(+\infty, \lambda)-\Gamma_{\rho, \tau}(-\infty, \lambda)\right\},
$$


where $n$ is any nonnegative integer. Let us now explore some consequences of this fundamental relation. Using the fundamental relation (12) for different choices of $\rho(x, \lambda)$ and $\tau(x, \lambda)$, we obtain the commutation relations given by $[6]$

$$
\left[I_{n}, A(\lambda)\right]=0, \quad\left[I_{n}, B^{\dagger}(\lambda)\right]=\frac{\hbar \lambda^{2 n}}{2^{n}} B^{\dagger}(\lambda) .
$$

By using the commutation relation (13b), it is easy to show that $I_{n}$ s satisfy eigenvalue equations like

$$
I_{n}\left|\mu_{1}, \mu_{2}, \cdots, \mu_{N}\right\rangle=\left(\frac{\hbar}{2^{n}} \sum_{j=1}^{N} \mu_{j}^{2 n}\right)\left|\mu_{1}, \mu_{2}, \cdots, \mu_{N}\right\rangle,
$$

where $\left|\mu_{1}, \mu_{2}, \cdots, \mu_{N}\right\rangle \equiv B^{\dagger}\left(\mu_{1}\right) B^{\dagger}\left(\mu_{2}\right) \cdots B^{\dagger}\left(\mu_{N}\right)|0\rangle$. Consequently, the commutation relation (13b) may be treated as the spectrum generating algebra for the quantum conserved quantities of DNLS model. Now we assume that the Bethe states $\left|\mu_{1}, \mu_{2}, \cdots, \mu_{N}\right\rangle$ represent a complete set of states in the corresponding Hilbert space. Thus two operators would coincide if they can be simultaneously diagonalised through these complete set of states and their eigenvalues always match with each other. Comparing (14) with (17), it is easy to find that

$$
\mathcal{C}_{0}=\frac{\alpha}{\hbar} I_{0}, \quad \mathcal{C}_{n}=\frac{2^{n+1}}{n \hbar} \sin (\alpha n) I_{n} .
$$

Thus the fundamental relation (12) not only yields the spectra of all $I_{n} \mathrm{~s}$, but also connect them with the conserved quantities which appear in the framework of QISM as expansion coefficients of $\ln \hat{A}(\lambda)$.

Next, we use the fundamental relation (12) to explicitly construct the first few quantum conserved quantities of DNLS model. Commutation relations between the Jost solutions and basic field operators play a crucial role in this construction. It is found that the first three quantum conserved quantities (number operator, momentum operator and the Hamiltonian) are given by [6]

$$
\begin{aligned}
& I_{0} \equiv N=\int_{-\infty}^{+\infty} \psi^{\dagger}(x) \psi(x) d x, \quad I_{1} \equiv P=-i \int_{-\infty}^{+\infty} \psi^{\dagger}(x) \partial_{x} \psi(x) d x, \\
& I_{2} \equiv H=\int_{-\infty}^{+\infty}\left\{-\psi^{\dagger}(x) \partial_{x x} \psi(x)+i \xi_{q}{\psi^{\dagger}}^{2}(x) \partial_{x} \psi^{2}(x)\right\} d x, \quad(16 a, b, c)
\end{aligned}
$$

where

$$
\xi_{q}=\frac{\xi}{\sqrt{1-\hbar^{2} \xi^{2}}} .
$$

It is worth noting that, due to the modification of coupling constant, the quantum Hamiltonian (16c) can not be expressed as normal ordered version of the corresponding classical Hamiltonian (2). Interestingly, the relation (17) between $\xi$ and $\xi_{q}$ is rather similar to the relation between rest mass and dynamical mass of a relativistic particle. Just as the dynamical mass of a relativistic particle coincides with 
its rest mass in the nonrelativistic limit, $\xi_{q}$ coincides with $\xi$ at $\hbar \rightarrow 0$ limit. In the ultrarelativistic limit, the dynamical mass of a particle tends towards infinity. In a similar way, $\xi_{q}$ can take arbitrary large value at $|\xi| \rightarrow \frac{1}{\hbar}$ limit. Consequently, even though QYBE restricts the value of $\xi$ as $|\xi| \leq \frac{1}{\hbar}$, there exists no such restriction on the value of corresponding quantum coupling constant $\xi_{q}$ (17). Thus the apparent limitation about the applicability of QISM in solving quantum DNLS Hamiltonian for the full range of its coupling constant is resolved in a very nice way.

\section{Conclusion}

By applying a variant of quantum inverse scattering method, we derive all possible commutation relations among the elements of the quantum monodromy matrix associated with DNLS model. These commutation relations enable us to construct the exact eigenstates for all conserved quantities of quantum DNLS model through algebraic Bethe ansatz. Next, we propose the fundamental relation for DNLS model. Using this fundamental relation, we are able to explicitly construct the quantum Hamiltonian and few other conserved quantities through basic field operators of this system. Surprisingly we find that, a new kind of coupling constant $\left(\xi_{q}\right)$, quite different from the classical one $(\xi)$, appears in the quantum Hamiltonian (16c) of the DNLS model. As a result, unlike the cases of most other integrable systems, this quantum Hamiltonian (16c) can not be obtained as normal ordered version of the corresponding classical Hamiltonian (2). Due to the presence of modified coupling constant in the quantum Hamiltonian, we are also able to consistently match various results of algebraic and coordinate Bethe ansatz in the case of DNLS model [6]. The $S$-matrix for two particle scattering and the distribution of single-particle momentum for quantum $N$-soliton states are two such examples where the results of algebraic and coordinate Bethe ansatz match with each other.

\section{References}

[1] Y.H. Ichikawa, K. Konno, M. Wadati and H. Sanuki: J. Phys. Soc. Jpn. 48 (1980) 279.

[2] Y. Kodama and A. Hasegawa: IEEE J. Quantum Electron QE-23 (1987) 510.

[3] U. Aglietti, L. Griguolo, R. Jackiw, S.-Y.Pi and D. Seminara: Phys. Rev. Lett. 77 (1996) 4406.

[4] A. Kundu and B. Basu-Mallick: J. Math. Phys. 34 (1993) 1052.

[5] B. Basu-Mallick and T. Bhattacharyya: Nucl. Phys. B 634 [FS] (2002) 611.

[6] B. Basu-Mallick and T. Bhattacharyya: Jost solutions and quantum conserved quantities of an integrable derivative nonlinear Schrodinger model, hep-th/0304063 to appear in Nucl. Phys. B.

[7] E.K. Sklyanin: in Yang-Baxter Equation in Integrable systems, Advanced series in Math. Phys. Vol. 10, edited by M. Jimbo (World Scientific, 1990) p.121.

[8] K. M. Case: J. Math. Phys. 25 (1984) 2306. 